\title{
Fabrication of Textured $\alpha$-SiC Using Colloidal Processing and a Strong Magnetic Field
}

\author{
Tohru S. Suzuki, Tetsuo Uchikoshi and Yoshio Sakka \\ Fine Particle Processing Group, Nano Ceramics Center, National Institute for Materials Science, Tsukuba 305-0047, Japan \\ In general, the mechanical and physical properties of a crystal depend on the direction of the crystal axis. The controlled development of \\ the crystallographic texture in ceramics is very useful for improvement of their properties. The preparation of the textured $\mathrm{SiC}$ polycrystal was \\ achieved by colloidal processing in a strong magnetic field. The $c$-axis of the $\mathrm{SiC}$ was parallel to the direction of the applied magnetic field. The \\ bending strength of the textured $\mathrm{SiC}$ depends on the crack-growth direction. [doi:10.2320/matertrans.MI200716]
}

(Received June 1, 2007; Accepted July 24, 2007; Published September 12, 2007)

Keywords: magnetic field, orientation, $\mathrm{SiC}$, colloidal processing

\section{Introduction}

Silicon carbide is one of the most important ceramics used as a structural and functional material in a wide variety of applications. Many studies have reported the control of its microstructure and improvement of the sinterability, mechanical properties and other properties using many kinds of additives. ${ }^{1-5)}$ Colloidal processing is generally able to control the dispersion of powders in a suspension and to produce a fine and dense microstructure. Several studies have reported that the sinterability and the mechanical properties of the $\mathrm{SiC}$ were improved by colloidal processing. ${ }^{6-10)}$

In general, the physical properties of a crystal depend on the direction of the crystal axis. Tailoring of the crystallographic texture in ceramics is very useful for the development of their properties. Many studies have reported the production of textured ceramics, such as by hot forging ${ }^{11)}$ and the Templated Grain Growth (TGG) method. ${ }^{12,13)}$ Application of the TGG method to the polycrystal $\mathrm{SiC}$ has also been reported. ${ }^{14)}$ The mechanical properties of SiC single crystals depended on the crystal planes. ${ }^{15)}$

More recently, the process of controlling the texture by a strong magnetic field has been reported for a variety of materials. ${ }^{16-21)}$ A strong magnetic field was applied to particles in stable suspensions during the consolidation process. The particles were rotated to an angle minimizing the system energy by a magnetic torque generated from the interaction between the magnetic anisotropy and the applied magnetic field. The magnetic torque, $T$, attributed to the interaction between the anisotropic susceptibility and the applied magnetic field is estimated using eq. (1). ${ }^{23,24)}$

$$
T=-\frac{\Delta \chi V B^{2}}{2 \mu_{0}} \sin 2 \theta
$$

where $\Delta \chi,\left(=\left|\chi_{/ /}-\chi_{\perp}\right|\right)$ is the anisotropy of the susceptibilities, which are measured in the direction parallel $\left(\chi_{/ /}\right)$and perpendicular $\left(\chi_{\perp}\right)$ to the $c$-axis in the tetragonal and the hexagonal crystal systems, $V$ is the volume of each particle, $\mu_{0}$ is the permeability in a vacuum, $B$ is the applied magnetic field and $\theta$ is the angle between the easy magnetization axis in a crystal and the imposed magnetic field direction. This is the driving force for magnetic alignment. The aligning direction of the crystal axis depends on the easy magnetization axis.
However, it is generally difficult to effectively apply a magnetic field in order to rotate fine diamagnetic particles, since fine particles tend to spontaneously agglomerate due to their strong attractive interactions (van der Waals forces). It is necessary to colloidally disperse the particles in a liquid in order to effectively utilize a magnetic field to rotate the particles due to reduction of the attractive interaction between the particles.

We demonstrate in this paper that control of the crystallographic texture in a polycrystalline $\mathrm{SiC}$ was achieved using the colloidal processing and a strong magnetic field, after which the bending strength was measured.

\section{Experimental Procedure}

A commercially available silicon carbide powder (OY-20, Yakushima Denko Co., Ltd., Japan) and alumina powder (TM-DAR, Taimei Chemicals Co., Ltd., Japan) with the average particle sizes of $0.55 \mu \mathrm{m}$ and $0.15 \mu \mathrm{m}$, respectively, were used as the starting materials. Aqueous suspensions of pH 10 were prepared that contained $30 \mathrm{vol} \%$ solids; the solids consisted of $\mathrm{SiC}$ that included 5 mass $\% \mathrm{Al}_{2} \mathrm{O}_{3}$ as a sintering aid. The $\mathrm{pH}$ of the suspensions was adjusted using tetramethylammonium hydroxide ( 25 mass $\%$ in methanol). The suspensions were ultrasonicated for $10 \mathrm{~min}$ and stirred for more than $4 \mathrm{~h}$. The suspensions were then consolidated by slip casting after an evacuation in a vacuum desiccator to remove as many air bubbles as possible. A strong magnetic field of $12 \mathrm{~T}$ was applied to the suspension during the slip casting at room temperature. The direction of the magnetic field was parallel to the casting direction. For comparison, some samples were prepared by slip casting without applying a magnetic field. The green compacts were densified isostatically so as not to disturbing the particle orientation by cold isostatic pressing (CIP) at $392 \mathrm{MPa}$ for $10 \mathrm{~min}$ and then isothermally sintered at $2473 \mathrm{~K}$ for $2 \mathrm{~h}$ in an Ar atmosphere under ambient pressure.

Aqueous suspensions of $\mathrm{SiC}$ containing both 5 mass $\%$ $\mathrm{Al}_{2} \mathrm{O}_{3}$ and 5 mass $\% \mathrm{Y}_{2} \mathrm{O}_{3}$ as the sintering additives were also prepared. The above-described $\mathrm{Al}_{2} \mathrm{O}_{3}$ and $\mathrm{Y}_{2} \mathrm{O}_{3}$ (C. I. Kasei Co., Ltd., Japan) with the average particle size of $0.03 \mu \mathrm{m}$ were used. The green $\mathrm{SiC}$ compacts were hot-pressed at $2173 \mathrm{~K}$ for $2 \mathrm{~h}$ at a pressure of $40 \mathrm{MPa}$ in an Ar atmosphere to 


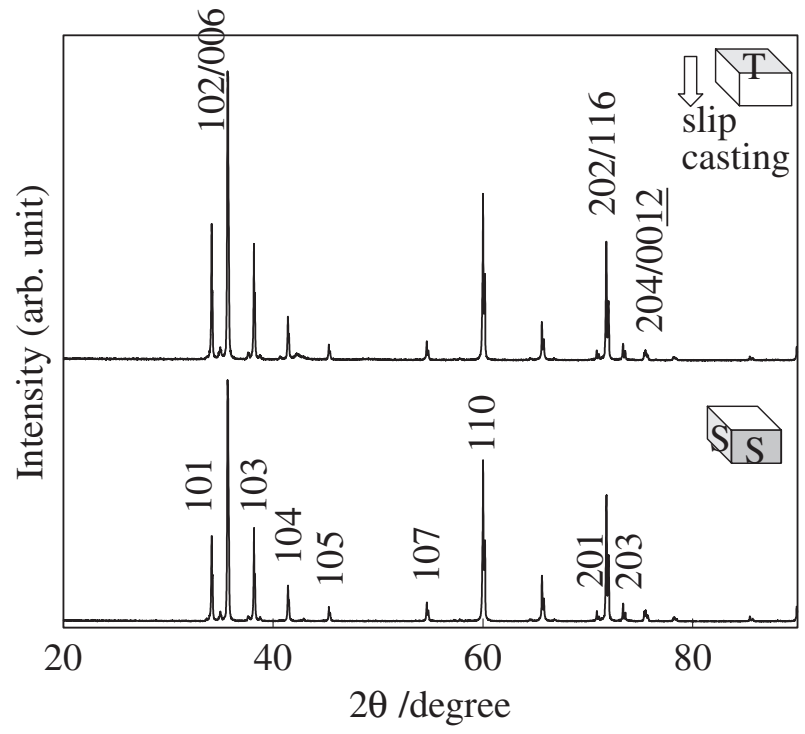

Fig. 1 X-ray diffraction patterns of SiC prepared by slip casting without applying a magnetic field, followed by pressureless sintering at $2473 \mathrm{~K}$ for $2 \mathrm{~h}$ in an Ar atmosphere.

produce a dense $\mathrm{SiC}$ polycrystal. The other experimental conditions of the hot-pressed sample were the same as that of the specimens prepared by the pressureless sintering.

The bending strength of the specimens, $3 \mathrm{~mm}$ in width and $3 \mathrm{~mm}$ in height, was measured at room temperature using the 3 -point bending test with a span length of $16 \mathrm{~mm}$ and a crosshead speed of $0.5 \mathrm{~mm} / \mathrm{min}$. In the textured sample the tensile surface was either parallel or perpendicular to the magnetic field. Each of the strength data of the textured and the untextured sample was an average of three measurements.

\section{Results and Discussion}

Figure 1 illustrates the X-ray diffraction (XRD) profiles of the specimen produced by slip casting without applying a strong magnetic field, followed by pressureless sintering at $2473 \mathrm{~K}$ for $2 \mathrm{~h}$ in an $\mathrm{Ar}$ atmosphere. The $\mathrm{T}$ and $\mathrm{S}$ planes are perpendicular and parallel to the casting direction, respectively. The specimen prepared without exposure to a magnetic field was confirmed to have a randomly oriented polycrystalline structure. By comparison, in the specimens exposed to a strong magnetic field, as shown in Fig. 2, the reflection at $2 \theta=35.6^{\circ}$ is extremely high in the VT-plane perpendicular to the applied magnetic field and the 110 reflection is every high in the VS-plane parallel to the magnetic field. The reflection at $2 \theta=35.6^{\circ}$ can be assigned to the Miller indices of 102 and 006. The inter-planar angle between the (102) plane and the basal plane is $70.6^{\circ}$, and the 006 plane is the basal plane. Considering that the 110 reflection perpendicular to the basal plane is high only in the VS-plane, the reflection at $2 \theta=35.6^{\circ}$ in the VT-plane can be attributed to almost only the 006 reflection. This demonstrates that a crystalline texture with the $c$-axis parallel to the magnetic field had been developed by colloidal processing in a strong magnetic field.

Figure 3 is SEM images of the cross-sectional surfaces of the specimen prepared with and without exposure to a

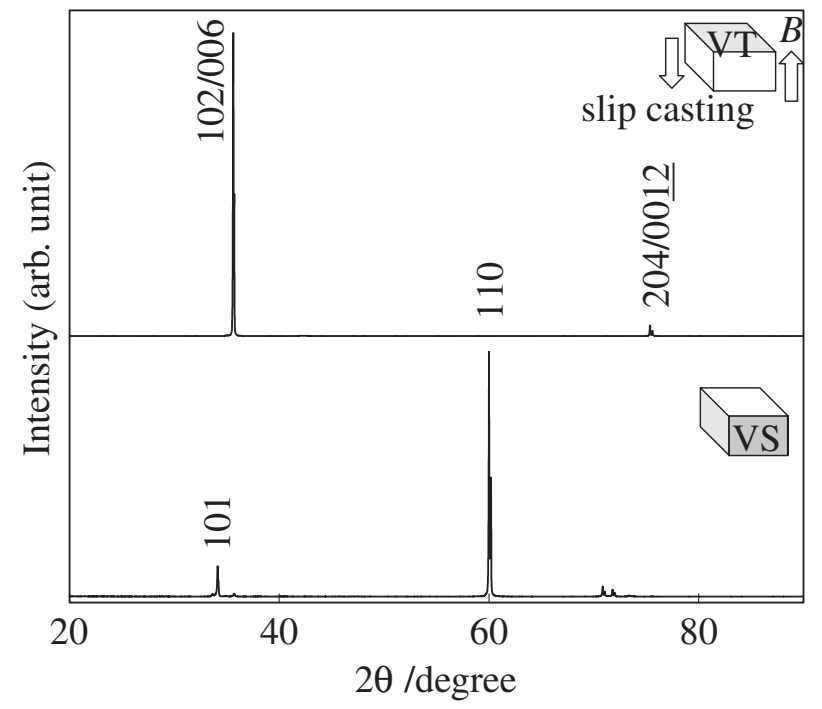

Fig. 2 X-ray diffraction patterns of $\mathrm{SiC}$ prepared by slip casting in a magnetic field of $12 \mathrm{~T}$, followed by pressureless sintering at $2473 \mathrm{~K}$ for $2 \mathrm{~h}$ in an $\mathrm{Ar}$ atmosphere.
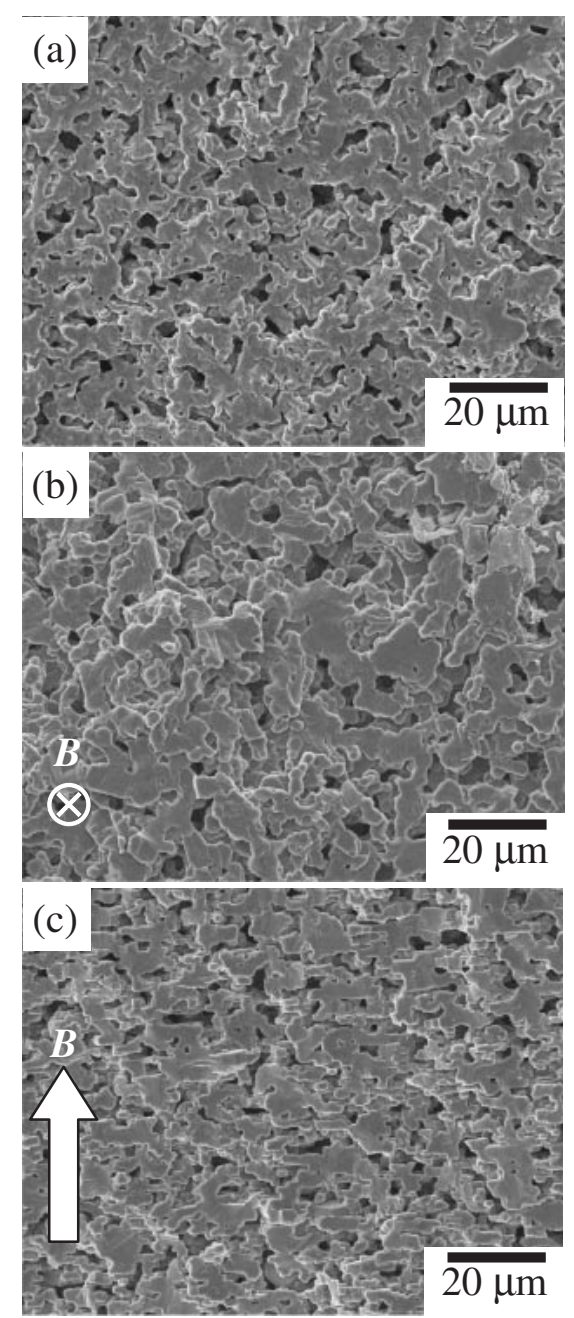

Fig. 3 SEM observation of cross-sectional surfaces of SiC prepared with and without a magnetic field, followed by pressureless sintering at $2473 \mathrm{~K}$ for $2 \mathrm{~h}$ in an Ar atmosphere: (a) the surface parallel to the casting direction without applying a magnetic field, (b) the surface perpendicular to the magnetic field, (c) the surface parallel to the magnetic field. 

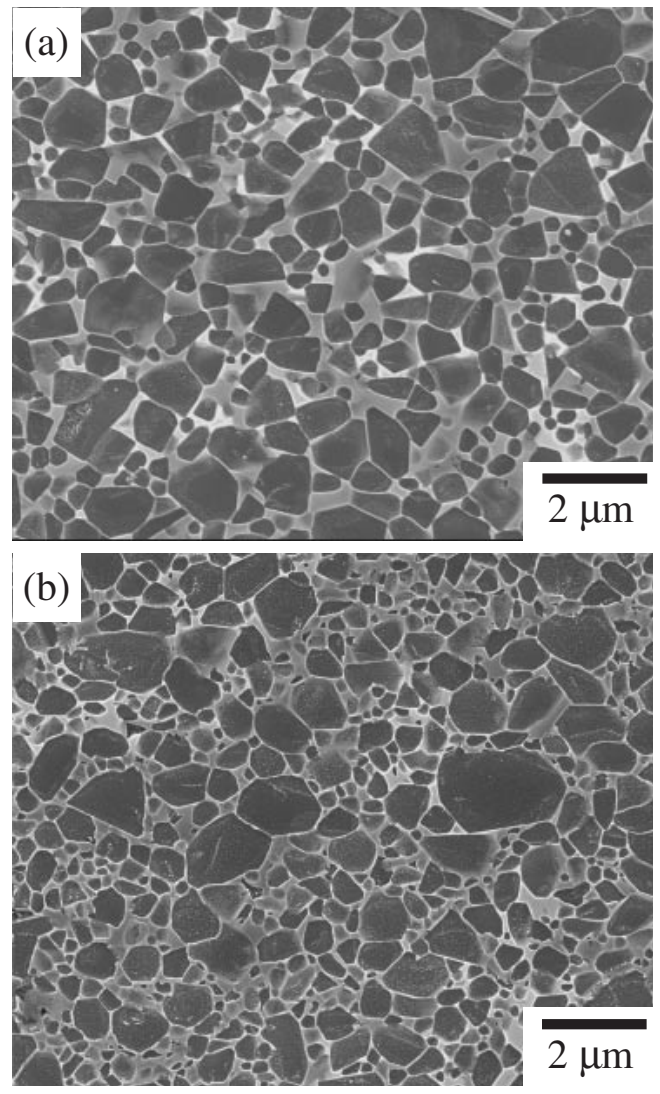

Fig. 4 Microstructure of the hot-pressed SiC prepared (a) without expose to a magnetic field and (b) with expose to a magnetic field.

magnetic field, followed by pressureless sintering at $2473 \mathrm{~K}$ for $2 \mathrm{~h}$ in an $\mathrm{Ar}$ atmosphere. There are many pores in both these specimens sintered by the pressureless sintering. The equiaxed grains appear randomly distributed in the untextured $\mathrm{SiC}$ prepared without a magnetic field, as shown in Fig. 3(a). Figs. 3(b) and (c) are the surfaces of the textured $\mathrm{SiC}$ perpendicular and parallel to the magnetic field, respectively. In Fig. 3 (b), the equiaxed grains are observed on the surface perpendicular to the magnetic field. By comparison, the slightly elongated grains are aligned perpendicular to the magnetic field in Fig. 3(c). Both these microstructural observations from the 2 directions show that the platelet grains are oriented perpendicularly relative to the magnetic field.

In order to densify the green $\mathrm{SiC}$ compacts, $\mathrm{Y}_{2} \mathrm{O}_{3}$ was additionally used as a sintering aid and the green compacts were sintered by hot-pressing. Figure 4 shows the microstructures of the $\mathrm{SiC}$ sintered at $2173 \mathrm{~K}$ for $2 \mathrm{~h}$ by hotpressing. Fig. 4(a) is the surface parallel to the casting direction in the $\mathrm{SiC}$ prepared without applying a magnetic field. Fig. 4(b) is the surface parallel to the magnetic field in the $\mathrm{SiC}$ prepared in a magnetic field. The dense microstructures were achieved in the hot-pressed samples, and both of the $\mathrm{SiC}$ prepared with and without applying a magnetic field consisted of equiaxed grains.

Figures 5 and 6 are the X-ray diffraction (XRD) profiles of the specimens sintered by hot-pressing. In Fig. 5, both of the profiles showed good agreement with each other, which were taken from the top and side surfaces, perpendicular and

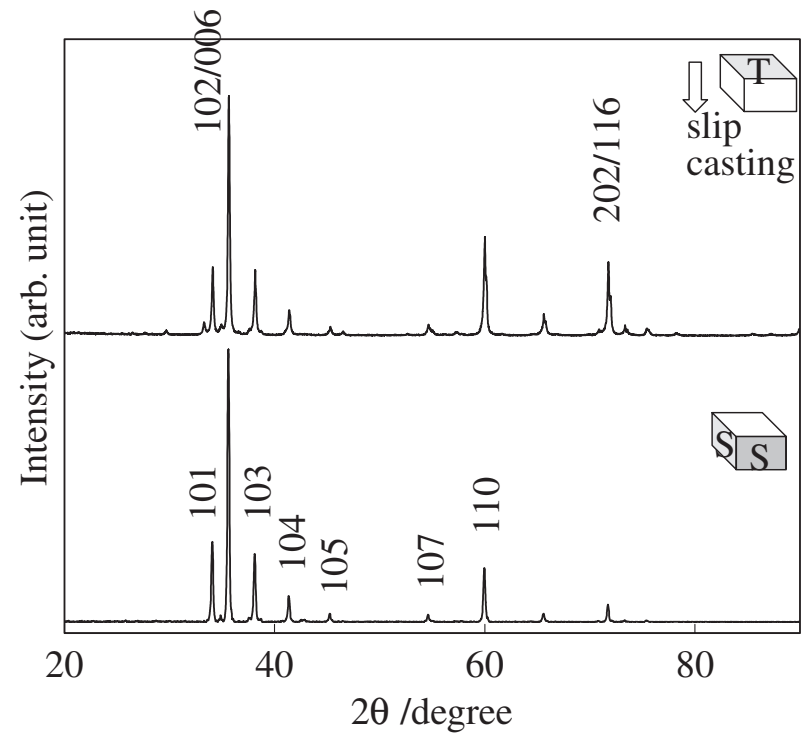

Fig. 5 X-ray diffraction patterns of SiC prepared by slip casting without a magnetic field, followed by hot-pressing at $2173 \mathrm{~K}$ for $2 \mathrm{~h}$ in an $\mathrm{Ar}$ atmosphere.

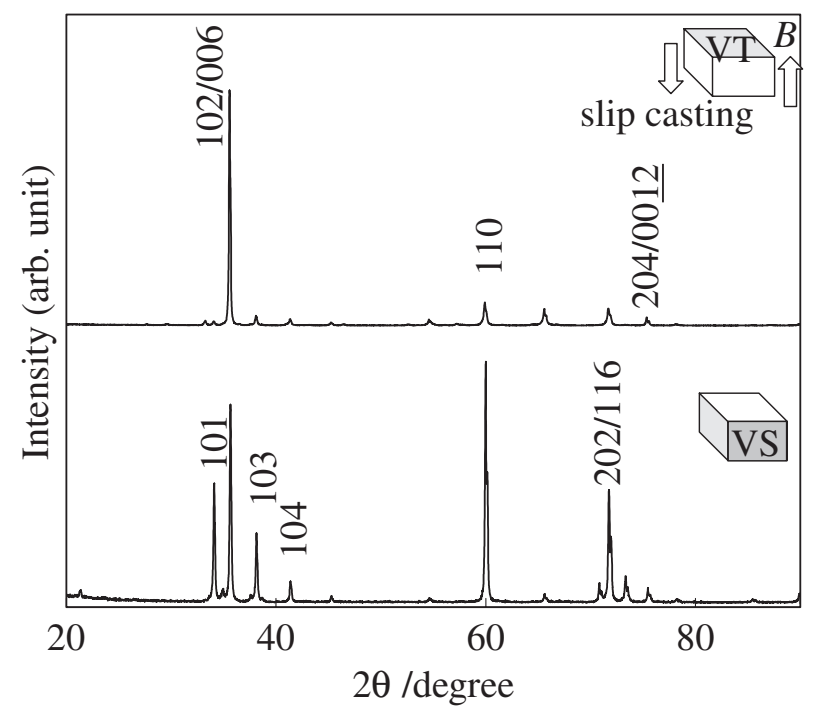

Fig. 6 X-ray diffraction patterns of $\mathrm{SiC}$ prepared by slip casting in a magnetic field of $12 \mathrm{~T}$, followed by hot-pressing at $2173 \mathrm{~K}$ for $2 \mathrm{~h}$ in an $\mathrm{Ar}$ atmosphere.

parallel to the casting direction without a magnetic field, respectively, this graph confirms that a silicon carbide sample prepared without using a magnetic field has a randomly oriented polycrystalline structure. This shows that the crystalline texture did not originate in the sintering by hotpressing. In Fig. 6, which shows the XRD of the surface perpendicular to the magnetic field (VT), the intensity of the reflection at $2 \theta=35.6^{\circ}$ is very high. In contrast, on the surface parallel to the magnetic field (VS), the intensity of the 110 reflection is very high. This XRD profiles show the same tendency of the specimen prepared by pressureless sintering at $2473 \mathrm{~K}$ (Fig. 2). Therefore, the crystalline texture in the hot-pressed sample was developed by the strong magnetic field, and we succeeded in producing a dense and textured $\mathrm{SiC}$. 

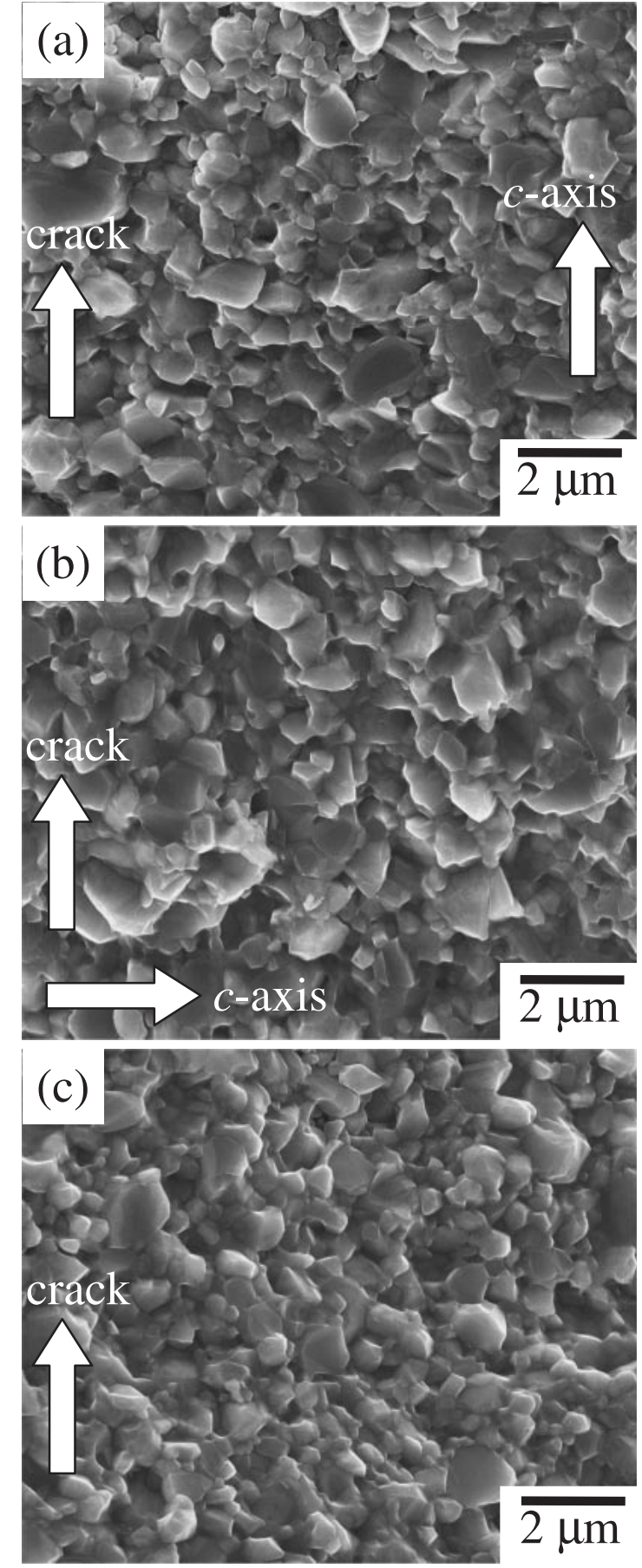

Fig. 7 SEM observation of fractured surfaces of $\mathrm{SiC}$ after the bending test: (a) crack propagation parallel to the $c$-axis, (b) crack propagation perpendicular to the $c$-axis, (c) the untextured $\mathrm{SiC}$.

However, a variety of reflections remained on the surface parallel to the magnetic field in the hot-pressed SiC (VS in Fig. 6), consequently, the degree of crystalline orientation of the hot-pressed $\mathrm{SiC}$ seems to be inferior to that of the $\mathrm{SiC}$ produced by the pressureless sintering at $2473 \mathrm{~K}$. This degradation in the orientation was attributed to the suppression of the grain growth due to the lower sintering temperature. We have reported for other systems that the development of the crystallographic orientation has a close relationship to the microstructure; i.e., grain growth improves the orientation degree. ${ }^{16-18,25)}$ As for another reason, the dispersion state could be changed in a suspension due to the additional use of $\mathrm{Y}_{2} \mathrm{O}_{3}$ particles as a sintering aid. Thus this dispersion state prevented the $\mathrm{SiC}$ particles from rotating in the suspension.

As regards the dense $\mathrm{SiC}$ prepared using $\mathrm{Al}_{2} \mathrm{O}_{3}$ and $\mathrm{Y}_{2} \mathrm{O}_{3}$ as the sintering additives and hot-pressing at $2173 \mathrm{~K}$ for $2 \mathrm{~h}$ at a pressure of $40 \mathrm{MPa}$, the 3-point bending strengths were 907 and $799 \mathrm{MPa}$ for the crack-growth directions parallel and perpendicular to the $c$-axis in the textured $\mathrm{SiC}$, respectively, and for the random $\mathrm{SiC}$, the strength was $724 \mathrm{MPa}$. The bending strength for the crack-growth direction parallel to the $c$-axis was higher than that perpendicular to the $c$-axis. The SEM observations of the fracture surfaces (Fig. 7) show that the fracture mode of the texture and the randomly oriented $\mathrm{SiC}$ was a mixture of intergranular (majority) and intragranular (minority) fractures regardless of the crack-growth direction. The deference between each fracture surface was not clear, thus it is difficult to explain the cause of the dependence of the strength. More research of this point needs to be done.

\section{Summary}

Control of the crystallographic orientation in polycrystalline $\mathrm{SiC}$ was achieved using a magnetic field, and the $c$-axis of the $\mathrm{SiC}$ crystal was parallel to the magnetic field. A dense and textured $\mathrm{SiC}$ can be produced using additional sintering aids and hot-pressing. Grain growth enhances the crystallographic orientation and the production of the platelet grains. The bending strength depends on the crack-growth direction.

\section{Acknowledgements}

The authors wish to thank Dr. Hidehiko Tanaka and Dr. Toshiyuki Nishimura of the National Institute for Materials Science for their helpful discussion, and Dr. Hideki Kakisawa for the hot-pressing. This study was partially supported by the Budget for Nuclear Research and of the Ministry of Education, Culture, Sports, Science and Technology, and also partially by the SHISEIDO Grants for Scientific Research and the Ishikawa Carbon Foundation.

\section{REFERENCES}

1) M. Omori and H. Takei: J. Am. Ceram. Soc. 65 (1982) C-92.

2) H. Tanaka, N. Hirosaki and T. Nishimura: J. Am. Ceram. Soc. 86 (2003) 2222-2224.

3) R. Yuan, J. J. Kruzic, X. F. Zhang, L. C. De Jonghe and R. O. Ritchie: Acta Mater. 51 (2003) 6477-6491.

4) Y. Zhou, K. Hirao, K. Watari, Y. Yamauchi and S. Kanzaki: J. Euro. Ceram. Soc. 24 (2004) 265-270.

5) K. Suzuki and M. Sasaki: J. Euro. Ceram. Soc. 25 (2005) 1611-1618.

6) Y. Hirata, N. Hidaka, H. Matsumura, Y. Fukushige and S. Sameshima: J. Mater. Res. 12 (1997) 3146-3157.

7) N. Hidaka, Y. Hirata, X. H. Wang and S. Tabata: J. Ceram. Soc. Japan 113 (2005) 143-148.

8) R. R. Rao, H. N. Roopa and T. S. Kannan: Ceram. Inter. 25 (1999) $223-$ 230.

9) L. M. Wang and W. C. Wei: J. Ceram. Soc. Japan 103 (1995) 434-443.

10) J. Zhang, M. Iwasa and D. Jiang: J. Am. Ceram. Soc. 88 (2005) $1013-$ 1016.

11) Y. Yoshizawa, M. Toriyama and S. Kanzaki: J. Am. Ceram. Soc. 84 (2001) 1392-1394. 
12) J. A. Horn, S. C. Zhang, U. Selvaraj, G. L. Messing and S. TrolierMckinstry: J. Am. Ceram. Soc. 82 (1999) 921-926.

13) T. Takeuchi, T. Tani and Y. Saito: Jpn. J. Appl. Phys. 39 (2000) $5577-$ 5580.

14) M. D. Sacks, G. W. Scheiffele and G. A. Staab: J. Am. Ceram. Soc. 79 (1996) 1611-1616.

15) H. Kitahara, Y. Noda, F. Yoshida, H. Nakashima, N. Shinohara and H. Abe: J. Ceram. Soc. Japan 109 (2001) 602-606.

16) T. S. Suzuki, Y. Sakka and K. Kitazawa: Adv. Eng. Mater. 3 (2001) 490-492.

17) T. S. Suzuki and Y. Sakka: Jpn. J. Appl. Phys. 41 (2002) L1272-L1274.

18) T. S. Suzuki and Y. Sakka: Chem. Lett. 31 (2002) 1204-1205.

19) T. S. Suzuki and Y. Sakka: Scripta Materialia 52 (2005) 583-586.
20) K. Inoue, K. Sassa, Y. Yokogawa, Y. Sakka, M. Okido and S. Asai: Mater. Trans. 44 (2003) 1133-1137.

21) A. Makiya, D. Kusano, S. Tanaka, N. Uchida, K. Uematsu, T. Kimura, K. Kitazawa and Y. Doshida: J. Ceram. Soc. Japan 111 (2003) 702704.

22) H. Kaga, Y. Kinemuchi, S Tanaka, A. Makiya, Z. Kato, K. Uematsu and K. Watari: J. Ceram. Soc. Japan 114 (2006) 1085-1088.

23) T. Sugiyama, M. Tahashi, K. Sassa and S. Asai: ISIJ Inter. 43 (2003) 855-861.

24) Y. Sakka and T. S. Suzuki: J. Ceram. Soc. Japan 113 (2005) 26-36.

25) T. S. Suzuki, Y. Sakka and K. Kitazawa: IEEE Trans. Appl. Supercond. 14 (2004) 1584-1587. 